\title{
ON EFFICIENTLY CREATING TEMPORALLY BIASED SAMPLES OVER DATA STREAMS: BEYOND MEMORYLESS FUNCTIONS
}

\author{
George Lagogiannis ${ }^{1}$ and Christos Makris ${ }^{2}$ \\ ${ }^{1}$ Department of Agricultural Economics and Rural Development, Agricultural University of Athens, Iera Odos 7511855 \\ Athens, Greece \\ ${ }^{2}$ Department of Computer Engineering and Informatics, University of Patras, 26504, Patras, Greece
}

\begin{abstract}
When creating a sample over a data stream, we sometimes need to give a priority to the recent past, over the distant one. This can be achieved if the probability of each stream element to exist in the sample decays as time passes, according to a bias-function. In order to obtain such a sample, one has to access some elements of the sample when a stream element arrives. For the exponential bias-function, according to which the probability of a sample-element to be deleted at each time-point, is the same for all sample elements, the number of needed accesses is $\mathrm{O}(1)$. For any other bias-function, one needs to access $\mathrm{O}(n)$ elements per incoming stream element, where $n$ is the sample-size. In this paper we present an algorithm that achieves $\mathrm{O}(1)$ amortized accesses for any bias-function such that at each time-point, the probability of a sample-element to be deleted increases over time.
\end{abstract}

\section{KEYWORDS}

Temporally Biased Sample, Stream, Markov Chains

\section{INTRODUCTION}

Massive amounts of data are being produced each second in the Internet (call logs, clicks, sensor readings e.t.c.). For the theoretical study of the problems related to the exploitation of these data, we use the data stream computational model, according to which data arrive continuously through a possibly infinite stream. The huge volume of the incoming data and our limited primary memory dictate that we can afford to store a small part of the stream only, which we call synopsis or sample of the stream. This sample can be used for the training of machine learning models that will then be assigned with the task of processing the incoming stream elements, aiming to extract useful information (in applications related to security, marketing, data management e.t.c.). A survey on stream sampling methods can be found in (Haas 2016) and (Aggarwal 2007).

However, data tend to change over time and the efficiency of machine learning models depends greatly on their ability to accordingly adapt. Static machine learning models lack this ability, but this problem can be solved through a periodically retraining process that allows such models to be used in dynamic settings. This approach was proposed in (Hentschel et al 2018) in the setting of graph analysis algorithms and has recently been adopted in the MacroBase system (Bailis P. et al 2017). The only available data for this retraining process are the elements of the sample and for the retraining to be successful, the recent history of the stream must occupy a large enough portion of the sample.

Assuming that the main memory can store $n$ stream elements, one could trivially include in the sample the $n$ most recent stream elements. But then the more distant past of the stream is totally ignored, and this fact may be a little extreme. A more "mild" approach would be to create a temporally biased sample where the stream element that arrived at time $r$ "survives" in the sample at time $t$ (where $t \geq r$ ) with probability proportional to a time-decay bias-function $f(r, t)$ which is defined to be monotonically decreasing with $t$ (for fixed $r$ ) and monotonically increasing with $r$ (for fixed $t$ ). We say that such a sample is consistent with function $f(r, t)$. 
In (Aggarwal 2006), an algorithm is introduced for creating a sample consistent with the exponential bias-function, $f(r, t)=e^{-\lambda(t-r)}$ where the parameter $\lambda$ defines the bias rate and typically lies in the range $[0,1]$ with very small values. The interesting property of this algorithm is that only one sample element is accessed each time a new stream element arrives. This element is the one to be replaced in the sample by the incoming stream element. It follows that the number of elements in the sample remains the same at all times, it is thus a reservoir sampling algorithm. Clearly, the number of accessed sample elements per stream element is a major indicator of the efficiency of any sampling algorithm. In the following we name a sampling algorithm time-efficient if it achieves $\mathrm{O}(1)$ accessed sample elements per stream element.

The exponential function "treats" all the elements in a memoryless approach, meaning that the probability of each stream element to exist in the sample drops according to the same rate as time passes, no matter how old the element is in regard to the current time-point. More formally, a bias-function $f(r, t)$ is memoryless if the fragment $f(r, t) / f(r, t-1)$ (referred in the following of the paper as $g(r, t))$ is the same for all $r, t(t \geq r)$. In (Aggarwal 2006) it was left open whether such a sample can be efficiently maintained for non memoryless functions, i.e. for functions $f(r, t)$ where $g(r, t)$ is not the same for all $\mathrm{r}, t(t \geq r)$.

Apparently, an algorithm that maintains such a sample for any given time-decay bias-function $f(r, t)$ is not difficult to be derived. According to Efraimidis (2010) there exist two such algorithms, i) an algorithm (referred as A-Chao) which had long ago been presented by Chao (1982) and ii) an algorithm (named A-ES) presented in (Efraimidis \& Spirakis 2006). Indeed both algorithms can solve the problem, they dictate however that the weights of the elements in the sample must be recomputed at each time-point and this fact leads to a time complexity of $\mathrm{O}(n)$ per incoming stream element. The open problem stated by Aggarwal (2006), is whether or not an algorithm can be created such that given any non-memoryless bias-function $f(r, t)$, the algorithm creates a sample consistent with $f(r, t)$ without the need to access all the sample elements, each time a new stream element arrives. Ideally, such an algorithm should access only $\mathrm{O}(1)$ elements of the sample, achieving the time-complexity of the algorithm presented by Aggarwal (2006).

In this paper we provide an insight to this open problem, by focusing on the time-complexity and dropping the reservoir constraint. Said otherwise, the sample may grow or shrink and we are not interested in providing probabilistic guarantees on its size. We only care about the time-efficiency of the algorithm, matching the time-efficiency of (Aggarwal 2006) in an amortized sense, creating thus an amortized efficient algorithm for non-memoryless functions.

\section{OUR RESULT}

We divide all non-memoryless functions into 2 categories: i) the harder to the older category, contains all the functions for which $g(r, t)$ decreases as $t-r$ increases and ii) the softer to the older category, contains all the functions for which $g(r, t)$ increases as $t-r$ increases. Given the type of time bias we need (i.e. the need to give priority to the recent past instead of the distant one), it follows that the "harder to the older" category is our target.

Let $M$ be the size of the available primary memory and $f(r, t)$ be a time-decay bias-function that belongs to the "harder to the older" category. We call $f(r, t)$ M-implementable if the average size of a sample consistent with $f(r, t)$ is at most equal to $M$. We present an amortized efficient algorithm that creates a sample consistent with any $M$-implementable time-decay bias-function $f(r, t)$ that belongs to the "harder to the older" category if:

- $0 \leq f(r, t) \leq 1$

- $f(t, t)=1$

- $g(r, t)>0.5$ for all $r, t$ such that $t \geq r$

The first two constraints make sense, however the third may raise suspicion that the pool of available functions is significantly reduced. This is not the case because if $g(r, t) \leq 0.5$ for all the elements in the sample, then each stream element is expected to survive in the sample for at most two time-points on the average. It seems that such a function is too hard to be of practical interest. Nevertheless, a function where $g(r, t)$ becomes 0.5 or less for very old elements (i.e. for elements having inclusion probability close to 0 ) is certainly of practical use because the third constraint can easily be put aside without changing the time-complexity of our algorithm. 
It must be made clear that our algorithm does not use a reservoir, meaning that the size of the sample is not kept unchanged during the execution of the algorithm. By "amortized efficient" we mean that the amortized number of sample elements accessed per stream element is $\mathrm{O}(1)$. Our algorithm requires a function that given $r, t$, returns $f(r, t)$. This is unavoidable since it works for any function following the above restrictions. In an amortized sense, it needs $\mathrm{O}(1)$ random numbers between 0 and 1 per incoming stream element. We adopt the framework given in (Aggarwal 2006) where time is defined by the arrival of the stream elements i.e. each time-point $t$ defines the (one) stream element that arrived at time $t$. An alternative approach adopted in (Hentschel et al 2018) would be to use wall clock times, allowing empty time-points (i.e. time-points when no stream element arrives) and batch insertions (i.e. time-points when more than one stream elements arrive). However, the framework of (Aggarwal 2006) suffices for showing the efficiency of our algorithm. To the best of our knowledge this is the first time that a sampling algorithm able to "achieve" any function that matches the above restrictions is presented.

\section{THE "TOOLS"}

\subsection{The Random Walk}

Our algorithm is based on a random walk which we shall describe in this subsection. Let us assume that we have $n$ sample elements $u_{1}, u_{2}, \ldots u_{\mathrm{n}}$, that have arrived (my means of a data stream) and been stored in a list according to their arrival time (i.e. each element has arrived after its neighbor on the left). The leftmost element $\mathbf{u}_{0}$ of the list is artificial, and has not arrived through the stream.

Each sample-element (i.e. each node of the list) defines a slot which contains two states in the random walk. In particular, if $u_{\mathrm{i}}(1 \leq \mathrm{i} \leq n)$ is an element of the sample, its slot contains the states $u_{\mathrm{i}}$-go and $u_{\mathrm{i}}$-select. A random walk performed by an entity which we call, cursor is defined on this list, as follows: The cursor starts from the leftmost slot and either moves to the slot on the right or jumps back to the leftmost slot. Each move (to the right) of the cursor between two consecutive slots, is called hop. The event $i \stackrel{t}{\longrightarrow} i+1$ is the event that a hop from slot $i$ to slot $i+1$ occurs at time $t$. The event $i \stackrel{t}{\longrightarrow} j$ is a sequence of $j$ - $i$ consecutive hops from slot $i$ to slot $j$ that occur at time $t$.

When the cursor reaches a slot, we toss a fair coin in order to decide on the state of the slot that the cursor will visit. As a result, when the cursor visits a slot, each of the two states of the slot have equal probability of being visited by the cursor. The only slot where we do not toss a coin is the one corresponding to the artificial element because it contains only one state, which is a go-state.

The reason for the introduction of 2-state slots, is the need for inserting randomness into the task of choosing the element to be deleted. In particular, if each element accessed by the cursor is deleted (with probability 1), then the elements are deleted in a left to right order, and the sample always contains the $n$ most recent elements of the stream. By setting an element candidate for deletion only if its select-state is accessed by the cursor, we introduce the necessary randomness for allowing the deletion of an element while older elements (than the one deleted) continue to exist in the sample.

The above described random walk on a list that contains 5 elements (plus the artificial one), is shown in Figure 1. In Figure 1, $p(i \stackrel{t}{\longrightarrow} i+1)$ is equal to the sum of the probabilities corresponding to the two straight solid (red) arrows leaving any of the two states associated with slot $i$. For example, by assigning to each straight solid arrow in Figure 2 a probability of 0.45 there is a probability of 0.9 for moving to the next slot. Then, by assigning a probability of 0.1 to each solid curved arrow, it follows that there is a 0.1 probability of moving to the leftmost slot from any slot other than the rightmost one. The thick curved dashed arrows connecting the rightmost states to the leftmost slot, correspond to transition probabilities equal to 1 . 


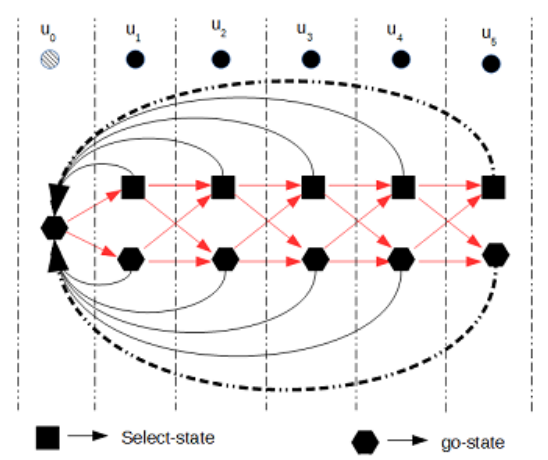

Figure 1. An example of the random walk upon which our algorithm is based

\subsection{Basic Structures and Notation}

Let $S$ be a possibly infinite data stream and $f(r, t)$ be a "harder to the older" bias-function. Our algorithm will create a sample that is consistent with $f(r, t)$, i.e. the stream element that arrived at time $r$ (denoted as $S(r)$ ) exists in the sample at time $t(t \geq r)$ with probability $p(r, t)=f(r, t)$. Time is defined by the incoming stream elements, meaning that $S(r)$ arrives at time $r$.

The elements of the sample are stored in a list sample-list, where the most recent element becomes the rightmost element of the list therefore the sample-elements are sorted according to their arrival times. We assume that the leftmost element of the sample-list was inserted in the sample at time minus infinity i.e. it exists in the sample with probability 0 and cannot be deleted. This may seem to be a contradiction because no element of the sample exists in the sample with probability 0. Keep in mind however that this is an artificial element used for symmetry reasons and does not really exist in the sample. In the following, we name the elements of the sample-list using their distance from the leftmost (artificial) element. This way, we name the artificial element $\mathrm{u}_{0}$, its right neighbor $u_{1}$ etc. Note that when element $u_{i}$ is deleted from the sample, element $u_{i+1}$ becomes $u_{i}, u_{i+2}$ becomes $u_{i+1}$ etc. Also note that the numbers do not play a role in the algorithm, and are only used for easing the description.

\section{CREATING A TEMPORALLY BIASED SAMPLE}

\subsection{Description of the Algorithm}

Each time a new stream element arrives, we increment time, we set the current time to be the arrival time of the element and we insert the new element in the rightmost position of the sample-list. Then we execute a step of the walk, and we define a step to be a "trip" of the cursor from the artificial element and back to it. This means that we increment time only when the cursor points to the artificial element and as a result, time remains frozen when the cursor walks the list rightwards. A sample element is deleted only if the cursor accesses its select-state, and when a slot (element) is accessed, each state of the slot has equal probability of being accessed. Let $r_{i}, r_{i+1}$ be the arrival times of elements stored in slots $\mathrm{i}$ and $\mathrm{j}$ respectively. We set $p(i \stackrel{t}{\longrightarrow} i+1)$ and $p(i \stackrel{t}{\longrightarrow} 0)$ as follows:

- $\left.\quad p(i \stackrel{t}{\longrightarrow} i+1)=\frac{1-g\left(r_{i+1}, t\right)}{1-g\left(r_{i}, t\right)}\right)$ if $i \neq 0$

- $\quad p(i \stackrel{t}{\longrightarrow} i+1)=2-2 * g\left(r_{i+1}, t\right)$ if $i=0$

- $p(i \stackrel{t}{\longrightarrow} 0)=1-p(i \stackrel{t}{\longrightarrow} i+1)$ 
To create the random event $i \stackrel{t}{\longrightarrow} i+1$ we first compute $p(i \stackrel{t}{\longrightarrow} i+1)$, through a function that given $r$, $t$ returns $f(r, t)$. Then we access a random number between 0 and 1 , and the event occurs if this random number is at least equal to $p(i \stackrel{t}{\longrightarrow} i+1)$.

Observe that when a new stream element arrives, the cursor always rests at the artificial element. The randomness introduced by the random walk involves the states accessed by the cursor during its traversal of the sample list, from the artificial element and back to it. A more formal description of the algorithm follows:

\section{Algorithm for creating a temporally biased sample}

$t \leftarrow t+1$

We insert the new stream element at the rightmost position of the sample-list (thus, when the first stream element arrives, it becomes the right neighbor of the artificial element).

delete_item $\leftarrow 0$

$$
\begin{aligned}
& j \leftarrow 0 \\
& \text { Repeat } \\
& k \leftarrow j
\end{aligned}
$$

We toss a biased coin with $p$ (heads $)=p(j \stackrel{t}{\longrightarrow} j+1)$

if "heads" occur

$j \leftarrow j+1$

if delete_item $=1$ then we delete element (slot) $k$ end if

We toss a fair coin.

If "heads" occur then delete_item $\leftarrow 1$ else delete_item $\leftarrow 0$ end if

else

$j \leftarrow 0$

end if

if delete item $=1$ then we delete element (slot) $k$ end if

Until $j=0$

\subsection{Analysis of the Algorithm}

We must prove that our random walk is valid. Clearly, the sum of the probabilities of all the events occurring in any state are equal to 1 by definition (see Subsection 4.1). It remains to show that all transition probabilities are between 0 and 1 .

Theorem 1: By setting the transition probabilities as shown in Subsection 4.1, all transition probabilities are between 0 and 1 .

Proof: If $i=0$, then $p(i \stackrel{t}{\longrightarrow} i+1)$ is valid because:

$p(i \stackrel{t}{\longrightarrow} i+1)<1 \$$ since $g\left(r_{1}, t\right)>0.5$ and $p(i \stackrel{t}{\longrightarrow} i+1) \geq 0$ since $g\left(r_{u_{1}}, t\right) \leq 1$

If $i \neq 0$, then $p(i \stackrel{t}{\longrightarrow} i+1) \geq 0$ because the enumerator and the denominator are both greater than 0 . In advance, observe that $r_{i+1}>r_{i}$ because $u_{i}$ is older than $u_{i+1}$. According to the definition of the "harder to the older" functions, it follows that $g\left(r_{i+1}, t\right)>g\left(r_{i}, t\right)$. This means that $1-g\left(r_{i+1}, t\right)<1-g\left(r_{i}, t\right)$ and as a result, $p(i \stackrel{t}{\longrightarrow} i+1)<1$.

Theorem 2: The algorithm creates a sample consistent with the bias-function $f(r, t)$.

Proof: Let us assume that element $u_{i},(1 \leq i \leq n)$ arrived at time-point $r_{i}$. Then at each time-point $t$ the cursor will reach the slot corresponding to element $u_{i}$ with probability:

$$
\begin{aligned}
p(0 \stackrel{\mathrm{t}}{\longrightarrow} i)=p(0 \stackrel{t}{\longrightarrow}) * p(1 \stackrel{t}{\longrightarrow} 2) * \ldots * p(i-1 \stackrel{\mathrm{t}}{\longrightarrow} i) \Rightarrow \\
\Rightarrow p(0 \stackrel{t}{\longrightarrow} i)=\left(2-2 * g\left(r_{1}, t\right)\right) * \frac{1-g\left(r_{2}, t\right)}{1-g\left(r_{1}, t\right)} * \ldots * \frac{1-g\left(r_{i}, t\right)}{1-g\left(r_{i-1}, t\right)} \Rightarrow
\end{aligned}
$$




$$
\Rightarrow p(0 \stackrel{\mathrm{t}}{\longrightarrow} 1)=2-2 * g\left(r_{i}, t\right)
$$

According to our algorithm, if the cursor reaches slot $i$, the states of slot $i$ have equal probabilities of being accessed. As a result, the probability that we reach state $u_{i}$-select during time-point $t$ is:

$$
p\left(u_{i}-\text { select }\right)=1-g\left(r_{i}, t\right)
$$

Reaching the $u_{i}$-select state, means that we delete $u_{i}$, at time-point $t$. Therefore, the probability that $u_{i}$ survives in the sample during time-point $t$ is equal to $g\left(r_{i}, t\right)$. This way it follows that an element that arrived at time $r$ continues to exist in the sample at time $t$ with probability:

$$
p(r, t)=g(r, r+1) * g(r, r+2) * \ldots * g(r, t)=f(r, t) \text { since } f(r, r)=1
$$

The sample may shrink or grow but on the average its size is expected to be equal to $\lim _{t \rightarrow \infty} \sum_{i=1}^{t} f(i, t)$ since (according to Theorem 2) the sample is consistent with $f(r, t)$. In Section 2 we have assumed that $f(r, t)$ is $M$-implementable, therefore the average sample-size is a value at most equal to the size of the available primary memory.

The expected time-complexity per stream element at time $t$ is proportional to the expected number of slots accessed by the cursor during time-point $t$. This number depends on the elements present in the sample at time $t$. More formally, given a time-point $t$ and a set $H=\left\{\mathrm{u}_{1}, \mathrm{u}_{2}, \ldots, \mathrm{u}_{\mathrm{n}}\right\}$ that contains all the elements in the sample at time $t$ so that $u_{i}(1<\mathrm{i} \leq \mathrm{n})$ has arrived after $u_{j} \forall j<i$, let $E L_{H, t}$ be the expected number of slots accessed by the cursor at $t$. Then:

$$
\begin{gathered}
E L_{h, t}=1+p(0 \stackrel{t}{\longrightarrow})+p(0 \stackrel{t}{\longrightarrow} 2)+\cdots+p(0 \stackrel{t}{\longrightarrow} n) \Rightarrow \\
\Rightarrow E L_{H, t}=1+\sum_{i=1}^{n}\left(2-2 * g\left(r_{i}, t\right)\right)
\end{gathered}
$$

Although the expected time complexity at time $t$ depends on the existing sample at time $t$, we can derive an estimation of the expected time per stream element through an amortization approach over all the possible samples at time $t$. In particular, the algorithm will create a sample where the $r$-th element of the stream (i.e., $S(r)$ ) exists in the sample at time $t$ with probability $f(r, t)$ and the cursor will reach $S(r)$ at time $t$ with probability $2-2 * g(r, t)$. We define $E L_{t}$ to be the expected number of slots accessed at time $t$, amortized over all possible samples having been created at time $t$. It follows that:

$$
E L_{t}=\sum_{i=1}^{t}(2-2 * g(i, t)) * f(i, t)
$$

and then the amortized expected time-complexity of our algorithm at time $t$ is equal to $O\left(\lim E L_{t}\right)$ The following theorem states that the amortized expected time-complexity of our algorithm is $\mathrm{O}(1)$.

Theorem 3: Let $f(r, t)$ be an $M$-implementable time-decay bias-function supported by our algorithm. Then, $\lim _{t \rightarrow \infty} E L_{t}=2$

Proof: The proof is an immediate consequence of Theorem 2. According to Theorem 2, the algorithm creates a sample consistent with $f(r, t)$. By definition, the average size of this sample is at most $M$ since $f(r, t)$ is $M$-implementable. Since the average sample-size is neither infinite nor 0 we conclude that in the long run, for each element that is inserted into the sample, an element is deleted from the sample (in any other case, the sample size would either grow to infinity or shrink to 0 ). Since at each time-point one element is inserted into the sample, we conclude that on the average one element is deleted per time-point. According to the algorithm, each accessed slot (element) is deleted with probability 0.5 . As a result, the amortized number of elements accessed per time-point is 2 . 


\section{EXPERIMENTS}

For the experiments we define two different families of functions, i) increasing decay rate exponential-like bias-functions and ii) linear bias-functions.

A function $f(r, t)$ belongs to the increasing decay rate exponential-like family if:

$$
g(r, t)=1-\frac{c *(t-r)}{2(t-r+1)}
$$

We name constant $c$, $(\mathrm{c} \leq 1)$ decay factor. The smaller the value of $c$, the bigger the sample. Clearly, $g(r, t)$ drops as $t-r$ increases (i.e. $f(r, t)$ belongs to the "harder to the older" category). If $t=r$, it follows that $g(r, t)=1$ i.e. the cursor is not allowed to reach the rightmost element of the list which means that $p(t, t)=1$. Furthermore: $\lim _{t-r \rightarrow \infty} g(r, t)=1-c / 2$ i.e. $g(r, t)>0.5$

Linear bias-functions are functions of the form $f(r, t)=1-(t-r)^{*} c$ where $c$ (the decay factor) is less than 1 . To our knowledge, such a function has never been used in the literature in the context of sampling over a data stream. It is obvious that $g(r, t)$ drops as $t-r$ increases (i.e. $f(r, t)$ belongs to the "harder to the older" category). If $t=r$, it follows that $g(r, t)=1$ and as a result, the cursor is not allowed to reach the rightmost element of the list (i.e. $p(t, t)=1$ ). The problem is that if the arrival time $r$ of an element is $c-1$ time-points away from the current time-point, it follows that $g(r, r+c-1)=0.5$ which cannot be supported by our algorithm. However, in practice this is not a real problem, since if $g(r, t)=0.5, f(r, t)$ is really small. In our experiments, we have chosen to set the forward probability equal to 1 for elements that arrived at least $c-1$ time-points away from the current time-point, forcing thus the cursor to visit each such element on every time-point. This means that each such element is expected to remain in the sample for two time-points. As a result, small inconsistencies may occur in regard to $f(r, t)$ in the area of inclusion probabilities that are very close to 0 , but the portion of the sample that is inconsistent with $f(r, t)$ is negligible. The number of additional elements to be accessed per time-point is not affected asymptotically, because we introduce $\mathrm{O}(1)$ additional elements per time-point.
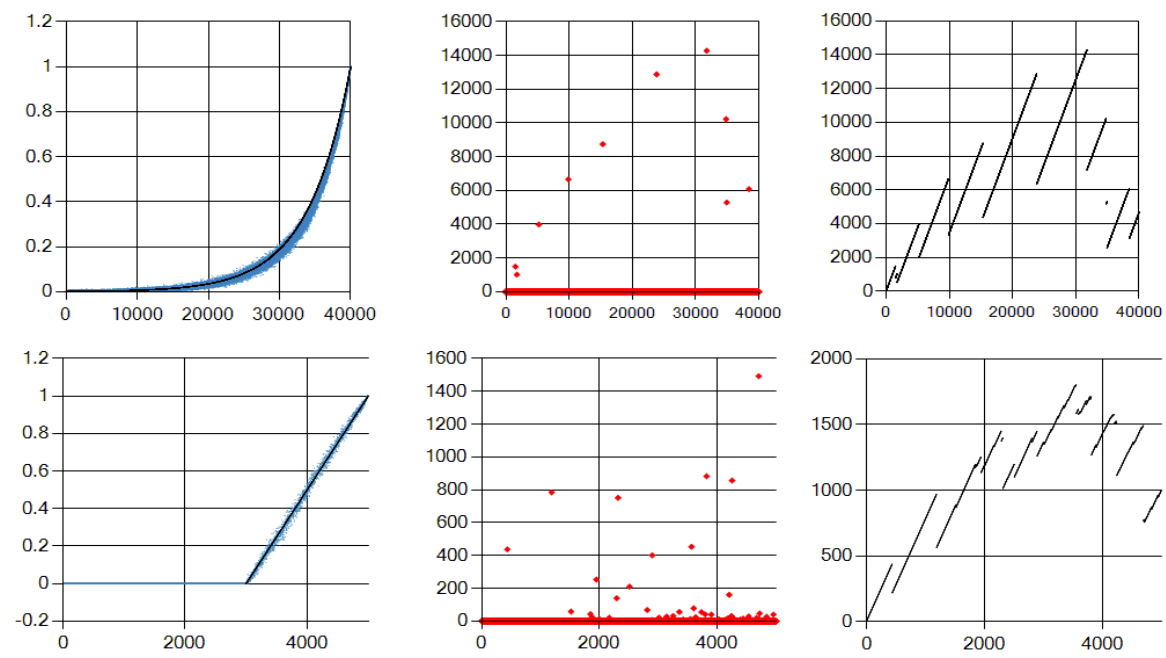

Figure 2. Experimental results

In Figure 2 we show the experimental results. In all of the graphs, the $\mathrm{x}$-axis contains the time-points. The upper three graphs correspond to the increasing decay exponential-like bias-function produced by setting $\mathrm{c}=1 / 3000$. The algorithm produces 500 samples, stops at 40000 time-points and $p(r, t)$ is experimentally computed (it is the number of samples containing $S(r)$ at time $t$, divided by the total number of samples). On the left graph, the black line corresponds to the theoretically calculated probability of each time-point to be represented in the sample at time $t=40000$, according to the given bias-function. The gray (blue) zone around the black line consists of very dense points, where each point corresponds to the experimentally 
calculated probability of the corresponding time-point to be represented in the sample. Clearly, our algorithm works as proved. For each time-point we measure the time spent by the algorithm by measuring the sample elements accessed during that time-point. The graph in the middle shows the time spent per time-point. Observe that most of the measured time costs are very close to the 0 since only a few sample elements were accessed. The amortized expected time was found to be 1.84, i.e. less than 2 and there is a simple explanation for that: In the beginning, there exists a large number of time-points when no sample element is accessed because $u_{1}$ is close to the current time-point and $p(0 \stackrel{t}{\longrightarrow} 1)$ is very small. If the algorithm is allowed to be executed for more time-points, the measured time-complexity is found to approach 2. Finally, the graph on the right shows the sample size at each time-point. It appears to be composed by distinct straight lines that can be explained as follows: Most of the time-points, no element is accessed and when that happens, the sample grows by one element. When finally the event $0 \stackrel{t}{\longrightarrow} 1$ occurs, it is very possible that the cursor performs a long walk over the sample-list, and each element accessed during this walk is deleted from the sample with probability 0.5 . Then the sample size drops significantly.

The lower three graphs in Figure 2 correspond to the linear function produced by setting $c=1 / 2000$. The algorithm produces 500 samples and stops at 5000 time-points. Each graph visualizes the same information as the graph above it. The left graph clearly shows that the produced sample is consistent with the given bias-function. The middle graph shows the time-cost per time-point and the one on the right shows the sample size. Each graph looks like the graph on top of it, because the "behavior" of our algorithm is similar for both functions.

\section{CONCLUSION}

We showed that given a time-decay bias-function $f(r, t)$ that belongs to the "harder to the older" category, a sample consistent with $f(r, t)$ can be maintained by sparing $\mathrm{O}(1)$ amortized time per incoming stream element. This result has been made possible through the use of random walks and shows the superiority of this technique over existing ones in terms of time-complexity. Furthermore, we consider our algorithm to be simple and easy to implement.

\section{REFERENCES}

Aggarwal C., 2006. On Biased Reservoir Sampling in the Presence of Stream Evolution. Proceedings of the 32nd International Conference on Very Large Data Bases (VLDB), Seoul, Korea, pp 607-618.

Aggarwal C. et al, 2007. A Survey of Synopsis Construction in Data Streams. In: Aggarwal C.C. (eds) Data Streams. Advances in Database Systems, vol 31, Springer, Boston, MA.

Bailis P. et al, 2017. MacroBase: Prioritizing Attention in Fast Data. Proceedings the ACM International Conference on Management of Data, Chicago, Illinois, USA, pp 541-556.

Chao, M. T., 1982. A general purpose unequal probability sampling plan. Biometrika, Vol. 69, No. 3 pp. 653-656.

Efraimidis P. S., Spirakis P. G., 2006. Weighted random sampling with a reservoir. Information Processing Letters, Vol. 97, No 5, pp 181-185.

Efraimidis P. S., 2010. Weighted random sampling over data streams. arXiv preprint arXiv:1012.0256.

Haas P. J., 2016. Data-Stream Sampling: Basic Techniques and Results. In Data Stream Management - Processing High-Speed Data Streams, Springer, 13-44.

Hentschel, B. et al, 2018. Temporally-Biased Sampling for Online Model Management. Proceedings of EDBT/ICDT 2018 Joint Conference, Vienna, Austria. 